\title{
Nicotinic acid modulates intracellular calcium concentration and disassembles the cytoskeleton
}

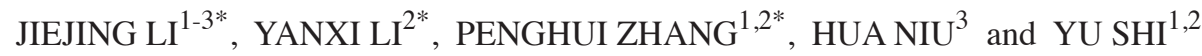 \\ ${ }^{1}$ Department of Clinical Laboratory, Children's Hospital of Chongqing Medical University; \\ ${ }^{2}$ Laboratory of Developmental Diseases in Childhood of Education Ministry, Key Laboratory of Pediatrics in Chongqing, \\ Chongqing International Science and Technology Cooperation Center for Child Development and Disorder, \\ Children's Hospital of Chongqing Medical University, Chongqing $400014 ;{ }^{3}$ Clinical Laboratory Centre, \\ The First People's Hospital of Yunnan Province, Kunming, Yunnan 650032, P.R. China
}

Received October 22, 2013; Accepted May 19, 2014

DOI: $10.3892 / \mathrm{mmr} .2014 .2576$

\begin{abstract}
Nicotinic acid (NA), a member of the vitamin B family, is well known for its functions in the treatment and prevention of atherosclerosis due to decreasing plasma levels of low-density lipoprotein cholesterol. In recent years, the major side effect of NA, cutaneous flushing, has also attracted extensive attention. However, the effects of NA in other aspects of physiology or cell biology have remained elusive. The present study provided evidence that high concentrations of NA were able to first reduce and later elevate intracellular $\left[\mathrm{Ca}^{2+}\right]$ in the NIH3T3 cell line. The reduction of the intracellular $\mathrm{Ca}^{2+}$ concentration was achieved within the initial $10 \mathrm{sec}$, and was preceded by a gradual elevation of intracellular $\left[\mathrm{Ca}^{2+}\right]$. Notably, marked accumulation of opaque materials in the perinuclear region was observed in NIH3T3 cells treated with $70 \mathrm{mM}$ NA. Further analysis revealed that treatment with $70 \mathrm{mM}$ NA for $1 \mathrm{~h}$ disassembled the microtubule and F-actin cytoskeleton systems and resulted in $\beta$-tubulin degradation in an ubiquitin-proteasome-dependent manner. These data indicated that high concentrations of NA disrupted cytoskeleton structures, which may have contributed to minus end (nucleus region) to plus end (cell membrane region)-directed transport processes and resulted in the deposition of material in the perinuclear region. Artificially increasing $\left[\mathrm{Ca}^{2+}\right]$ adding $\mathrm{CaCl}_{2}$ to the culture media effected the disassembly of F-actin, while it had no apparent effect on microtubules. These results suggested that the disruption of the cytoskeleton systems was not entirely due to the NA-induced elevation of $\left[\mathrm{Ca}^{2+}\right.$. Finally, microinjection of NA into xenopus embryos blocked the transport of melanosomes to the peripheral cellular area. In conclusion, the present
\end{abstract}

Correspondence to: Dr Yu Shi, Department of Clinical Laboratory, Children's Hospital, Chongqing Medical University, 136 Zhongshan ER Road, Chongqing 400014, P.R. China

E-mail: shiyu@cqmu.edu.cn

*Contributed equally

Key words: nicotinic acid, calcium, cytoskeleton, xenopus study indicated that NA disassembles F-actin and microtubule systems, thereby blocking cytoskeleton-dependent intracellular transport.

\section{Introduction}

The milestone results reported by Altschul et al (1) >50 years ago demonstrated that nicotinic acid (NA) has the capacity to decrease plasma lipids. As a result, this water soluble vitamin B family member has been widely used clinically for the treatment and prevention of atherosclerosis and other lipid-metabolic disorders $(2,3)$. At present, NA is one of the most effective agents that offers protection against cardiovascular risk factors by increasing high density lipoprotein (HDL) levels, while simultaneously decreasing very low density lipoprotein (VLDL) and low density lipoprotein (LDL) levels (4). The major side effect of NA is cutaneous vasodilatation, also known as 'flush', which limits its clinical utility and applications (5). NA functions by downregulating intracellular cyclic adenosine monophosphate (cAMP), the major intracellular mediator of prolipolytic stimuli, and subsequently decreases cellular levels of free fatty acids (5). Notably, prostaglandin has been demonstrated to have a vital role in flushing (6,7). Anti-lipid and flush effects are mediated by its G protein-coupled receptor GPR109A $(8,9)$. Despite extensive studies in the field of lipid metabolism, the effects of NA on other aspects of cellular physiology remain elusive. Previously, several groups have demonstrated that NA elevates intracellular $\left[\mathrm{Ca}^{2+}\right]$ in neutrophil (10), macrophage (8) and CHOK1 cell lines (9) in a GPR109A-dependent manner. Elevation of intracellular $\left[\mathrm{Ca}^{2+}\right]$ may transduce a number of different signaling pathways in different cell types. In the present study, variations in intracellular $\mathrm{Ca}^{2+}$ levels were observed under incubation with different concentrations of NA, and long-term $(1 \mathrm{~h})$ effects on the NIH3T3 cell line and its cytoskeleton were analyzed.

\section{Materials and methods}

Cell culture. CHO-K1 cells (cat.no CCL-61; American Type Culture Collection; Manassas, VA, USA) were grown in F12 medium (11765047; Life Technologies, Grand Island, NY, USA) supplemented with $10 \%$ fetal calf serum (FCS; 16170086; 


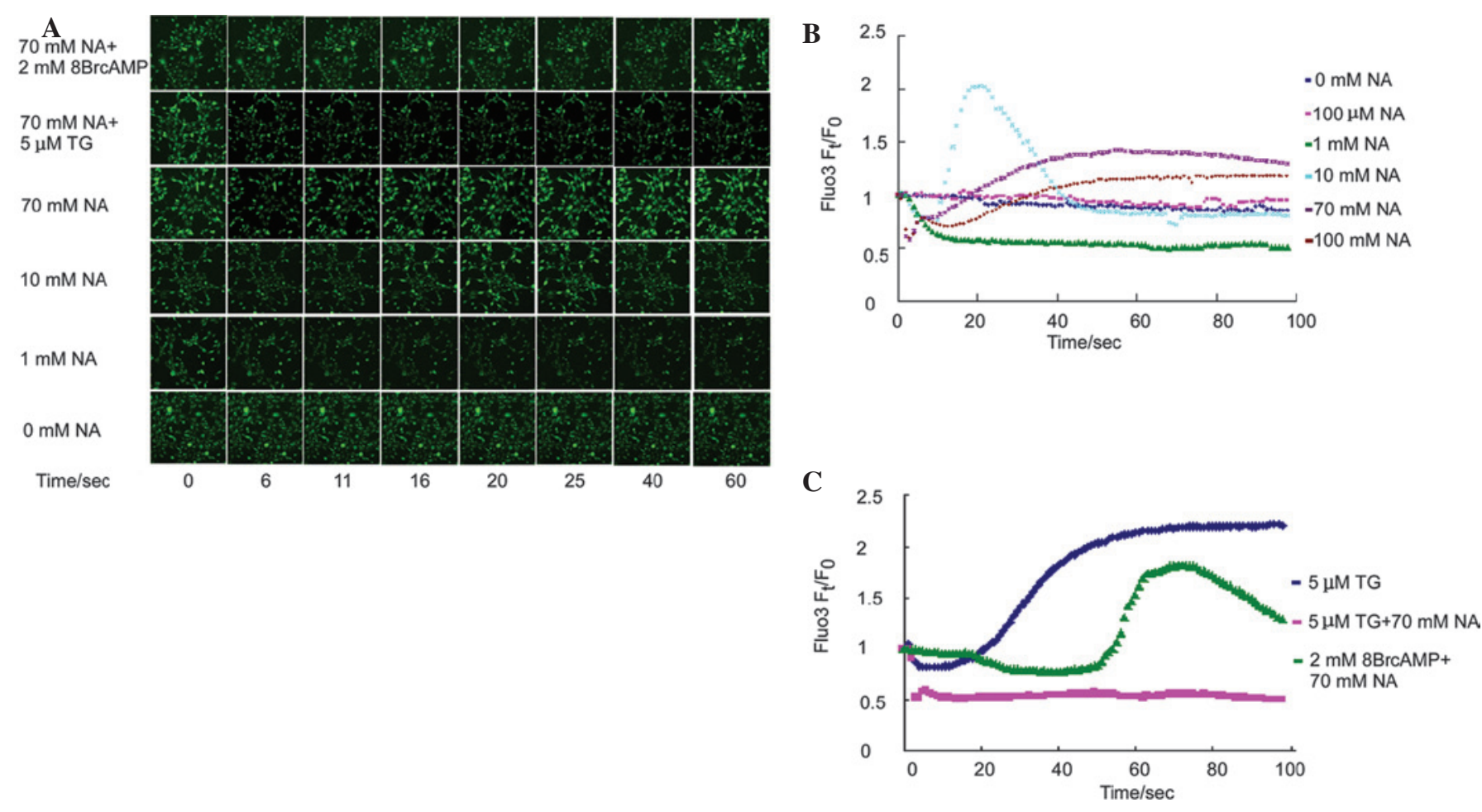

Figure 1. Time lapse assessment of intracellular $\left[\mathrm{Ca}^{2+}\right]$ levels upon exposure to different concentrations of NA. (A) Visualization of intracellular [Ca $\left.{ }^{2+}\right]$ upon exposure to different concentration of nicotinic acid and other drugs by staining with Fluo3 acetoxymethyl $\mathrm{Ca}^{2+}$ indicator; (B) time lapse statistical curve of intracellular $\mathrm{Ca}^{2+}$ levels upon exposure to different NA concentrations; (C) TG inhibits the elevation of [Ca $\left.{ }^{2+}\right]$ induced by NA (pink curve) and cAMP analog 8-Br-cAMP delays and alleviates NA-induced primary $\left[\mathrm{Ca}^{2+}\right]$ decrease (yellow curve). $\mathrm{F}_{0}$ : Fluo3-labeled intensity prior to exposure to NA or other drugs; $\mathrm{F}_{\mathrm{t}}$ : Fluo3-labeled intensity at time-point t. NA, nicotinic acid; TG, thapsigargin.

Life Technologies). The 293T cells (CRL-3216; American Type Culture Collection) were grown in Dulbecco's modified Eagle medium (DMEM; 12430047; Life Technologies) with $10 \%$ fetal calf serum (FCS). The NIH3T3 cells (CRL-1658; American Type Culture Collection) were cultured in DMEM with $10 \%$ FCS.

Time lapse measurement of intracellular $\left[\mathrm{Ca}^{2+}\right]$. The cells ( $2 \times 10^{4} /$ well) were allowed to adhere to a sterile 96-well cell culture plate (Greiner Bio-One) and incubated with Fluo3 acetoxymethyl (AM) $\mathrm{Ca}^{2+}$ indicator (Molecular Probes, Invitrogen Life Technologies, Carlsbad, CA, USA) for $1 \mathrm{~h}$ at $37^{\circ} \mathrm{C}$. The $\mathrm{Ca}^{2+}$ levels were assessed by measuring the fluorescent intensity using a Zeiss LSM 510 META confocal microscope and Zeiss Lsm Image Examiner software (FV10-ASW 2.1 Viewer; Carl Zeiss, Jena, Germany) was applied for quantitative analysis.

Fluorescent immunohistochemistry. The cells were fixed for 10 min with $3.7 \%$ paraformaldehyde (Sigma, St. Louis, MO, USA) and permeabilized with $0.2 \%$ Triton X-100 (Sigma). The F-actin stress fibers were labeled with Texas Red-X phalloidin (Molecular Probes, Invitrogen Life Technologies). The microtubule filaments were stained with monoclonal mouse anti- $\beta$-tubulin antibody (1:200; E1C-601; EnoGene, New York, NY, USA) and the secondary antibody was goat-anti-mouse-fluorescein isothiocyanate (1:200; Sigma).

Western blot analysis. The cells were incubated with MG132 (10 $\mu \mathrm{M}$; Santa Cruz Biotechnology, Inc., Santa Cruz, CA,
USA) and/or NA and collected at the appropriate time. The cells were boiled at $100^{\circ} \mathrm{C}$ in Lämmli buffer for $5 \mathrm{~min}$. The following antibodies were used: Mouse anti- $\beta$-tubulin monoclonal antibody (1:10,000; EnoGene E1C-601; EnoGene); mouse anti- $\beta$-actin monoclonal antibody $(1: 10,000$; ab6276; Abcam, Massachusetts, MA, USA); rabbit anti-H3 polyclonal antibody (1:10,000; H0164; Sigma); horseradish peroxidase (HRP)-goat anti mouse antibody (1:10,000; A3673; Sigma); HRP-goat anti-rabbit antibody (1:10,000; sc-2030; Santa Cruz Biotechnology, Inc.).

Xenopus embryo manipulation and microinjection. In vitro embryo fertilization and culture were conducted as described previously (11). For each embryo, $70 \mathrm{ng}$ of NA was injected at the 2 cell stage. The microinjection procedure was performed as described previously (12). The use of Xenopus embyos in the study was approved by the Ethics Comittee of the Children's Hospital of Chongqing Medical University (Chongqing, China).

\section{Results}

$N A$ regulates intracellular $\mathrm{Ca}^{2+}$ mobilization. To examine the time lapse effect of NA on intracellular free $\mathrm{Ca}^{2+}$ mobilization, Fluo3-labeled NIH3T3 cells were incubated with different concentrations of NA, and the fluorescence intensity was simultaneously assessed over $100 \mathrm{sec}$. The fluorescence intensity reflected the intracellular free $\mathrm{Ca}^{2+}$ concentration. Previous studies have demonstrated that $100 \mu \mathrm{M}$ NA induced transient intracellular $\left[\mathrm{Ca}^{2+}\right]$ elevation in CHO-K1 cells (9), 

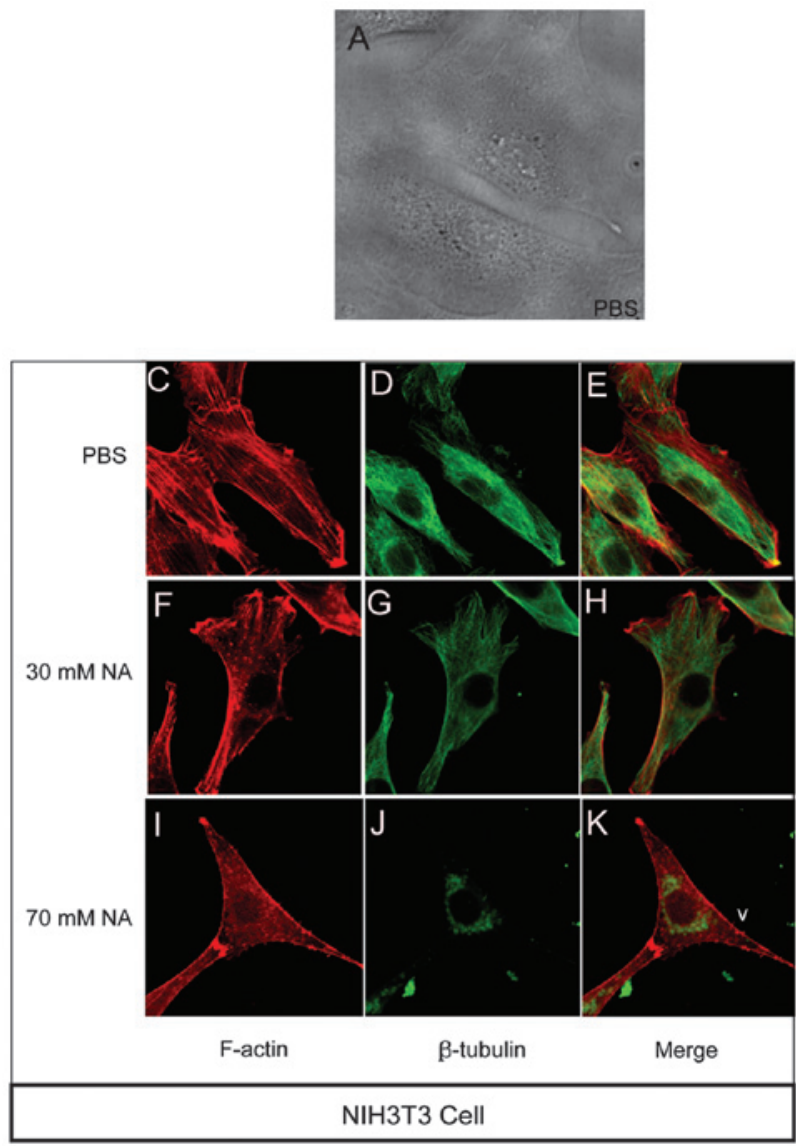
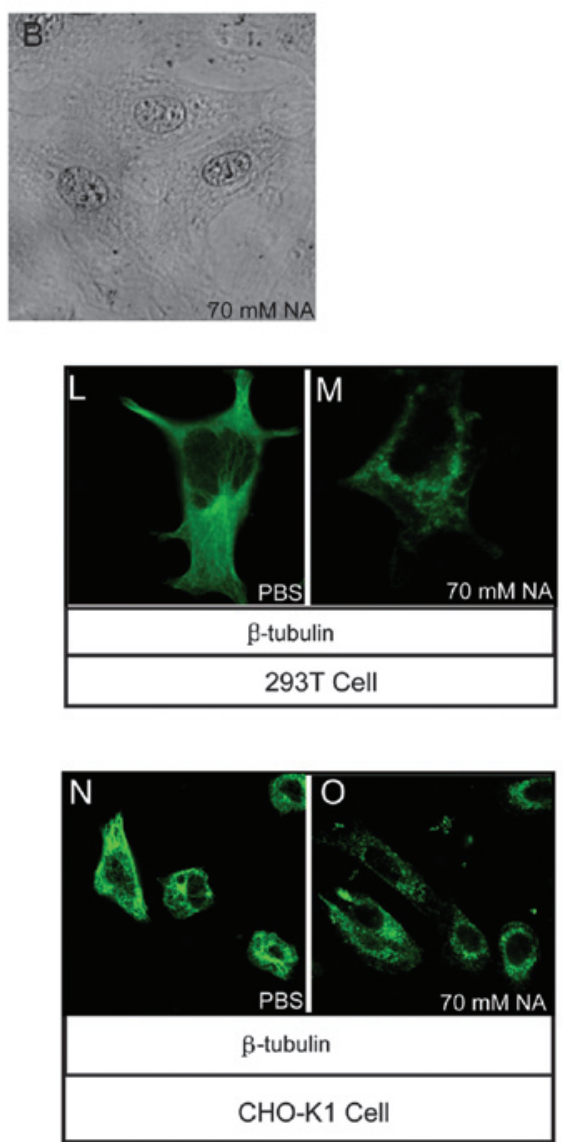

Figure 2. NA disassembles the cytoskeleton and deposits opaque materials at the peri-nuclear region. Compared with (A) the PBS-incubated control group, (B) $70 \mathrm{mM}$ NA results in evident nucleolar accumulation of opaque materials at peri-nucleus regions in NIH3T3 cells. In the PBS-incubated NIH3T3 cells, (C) F-actin and (D) microtubules are filament-rich as is apparent in (E) the merged image. Exposure to $30 \mathrm{mM}$ NA for $1 \mathrm{~h}$ partially disassembled (F) F-actin and (G) microtubules as is apparent in $(\mathrm{H})$ the merged image. $70 \mathrm{mM}$ NA completely disassembled (I) F-actin and (J) microtubules as apparent in (K) the merged image. Microtubule structure was disassembled when exposed to $70 \mathrm{mM} \mathrm{NA}$ for $1 \mathrm{~h}$ in both (L and M) 293T cells and (N and O) CHO-K1 cells. (Magnification, $\mathrm{x} 250$ ). NA, nicotinic acid.

macrophages (8) and matured neutrophils (10) within one to several minutes. In the NIH3T3 cell line, $100 \mu \mathrm{M}$ NA did not alter the intracellular $\mathrm{Ca}^{2+}$ mobilization (Fig. 1B, pink curve). The NIH3T3 cells were further exposed to a wider span of NA concentration gradients and the $\mathrm{Ca}^{2+}$ mobilization was assessed. At a $1 \mathrm{mMNA}$, the intracellular $\mathrm{Ca}^{2+}$ levels decreased by $50 \%$ within $10 \mathrm{sec}$ and no elevation was detected during the entire process (Fig. 1A and B, green curve). In the $10 \mathrm{mM}$ NA exposure group, intracellular free $\mathrm{Ca}^{2+}$ reduced precipitously similarly to the observations at $1 \mathrm{mM} \mathrm{NA}$; however, a transient sharp elevation-reduction n-turn like curve of $\mathrm{Ca}^{2+}$ mobilization was observed (Fig. 1A and B, light blue curve). Consistent with the $10 \mathrm{mM}$ group, both $70 \mathrm{mM}$ (Fig. 1B, purple curve) and $100 \mathrm{mM}$ (Fig. 1B, brown curve) NA decreased intracellular free $\left[\mathrm{Ca}^{2+}\right]$ within the first several seconds, and secondarily, triggered an elevation in intracellular free $\left[\mathrm{Ca}^{2+}\right]$. Of note, secondary increase in $\left[\mathrm{Ca}^{2+}\right]$ was slower with increasing NA concentration. In addition, NA-induced secondary $\left[\mathrm{Ca}^{2+}\right]$ was inhibited by thapsigargin (TG; Fig. $1 \mathrm{~A}$ and $\mathrm{C}$, pink curve), an endoplasmic reticulum (ER) $\mathrm{Ca}^{2+}$-ATPase pump inhibitor, which induces $\mathrm{Ca}^{2+}$ release from the ER. Furthermore, the NA-induced decrease in primary intracellular $\left[\mathrm{Ca}^{2+}\right]$ was delayed by the addition of $2 \mathrm{mM}$ of the cAMP analog 8Br-cAMP (Fig. 1A and C, green curve). These data suggested that the reduction of cAMP levels by NA may be responsible for the primary transient ER decrease and $\mathrm{Ca}^{2+}$ release by the endoplasmic reticulum (ER) contributed to the later observed $\mathrm{Ca}^{2+}$ elevation.

NA disassembles the cytoskeleton and deposits opaque materials at the perinuclear region. Besides intracellular $\mathrm{Ca}^{2+}$ wave variation, the results revealed that an accumulation of unidentified opaque material at the perinuclear region, forming a ring-type structure, as well as at the nucleolus, was markedly evident in the NIH3T3 cells following incubation with $70 \mathrm{mM}$ NA (Fig. 2B). However, in the phosphate-buffered saline (PBS)treated control group, the NIH3T3 cells exhibited a spread morphology and no perinuclear ring or dim nucleolus phenotypes (Fig. 2A). The highly visible nucleolus in the NA-treated cells suggested the activation of synthetic processes; however, the manner in which the perinuclear opaque rings formed remains elusive. Cytoskeletal organization has a number of important roles in intracellular transport processes. The assembly-disassembly homeostasis of F-actin and microtubules are regulated by numerous factors, including variations in intracellular $\left[\mathrm{Ca}^{2+}\right]$ (13-17). The NA-induced phenotypes identified in the present study allowed for the following hypothesis: NA changes intracellular $\left[\mathrm{Ca}^{2+}\right]$, thereby obstructing cytoskeletal 


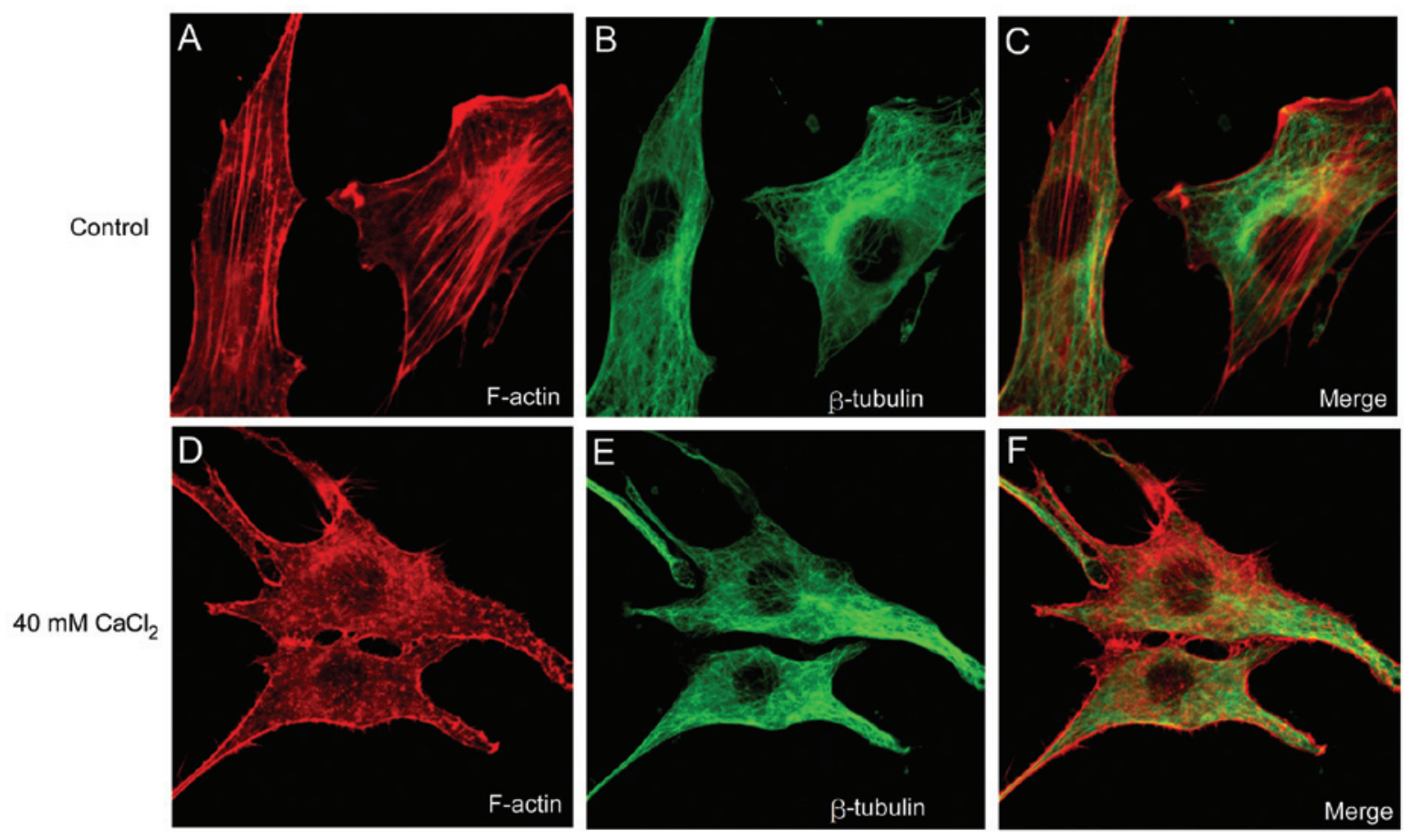

Figure 3. External addition of $\mathrm{CaCl}_{2}$ to the culture media and $1 \mathrm{~h}$ incubation disrupted the F-actin filaments, stained with Texas Red-X phalloidin, but did not affect the microtubules, stained with fluorescein isothiocyanate-conjugated antibody. (Magnification, x250).

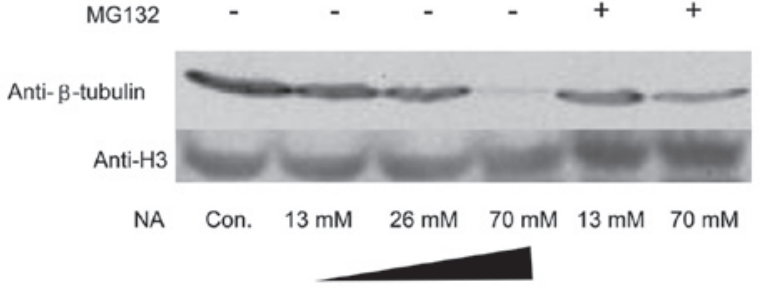

Figure 4. NA caused microtubule component $\beta$-tubulin degradation in an ubiquitin-proteasome pathway. NA, nicotinic acid.

integrated organization, then affecting cytoskeleton-dependent intracellular transport and finally causing the accumulation of material at the perinuclear area, forming an opaque ring like structure. To confirm this hypothesis, F-actin and microtubule structures were observed with Texas Red-X phalloidin and anti- $\beta$-tubulin antibodies, respectively, under different concentrations of NA. In the PBS-treated control group, the F-actin (Fig. 2C) and microtubules (Fig. 2D) were normally patterened, as shown in the merged image in Fig. 2E. The F-actin (Fig. 2F) filaments began to disassemble, forming punctuated spots and the microtubules (Fig. 2G) exhibited weaker staining following treatment with $30 \mathrm{mM} / 1 \mathrm{~h} \mathrm{NA}$. Following incubation with $70 \mathrm{mM} / 1$ h NA, the F-actin (Fig. 2I) and microtubule (Fig. 2J) cytoskeletons (merged in Fig. 2K) were completely disassembled, and the liberated microtubule residues accumulated at the distal end of the filopodia and perinuclear region (Fig. 2J). Further analysis confirmed the occurrence of microtubule disassembly with $70 \mathrm{mM}$ NA in the 293T (Fig. 2L and M) and $\mathrm{CHO}-\mathrm{K} 1$ cell lines (Fig. $2 \mathrm{~N}$ and $\mathrm{O}$ ). These data indicated that NA dissociates the F-actin and microtubule cytoskeleton, which may affect intracellular transport in a dose-dependent manner.

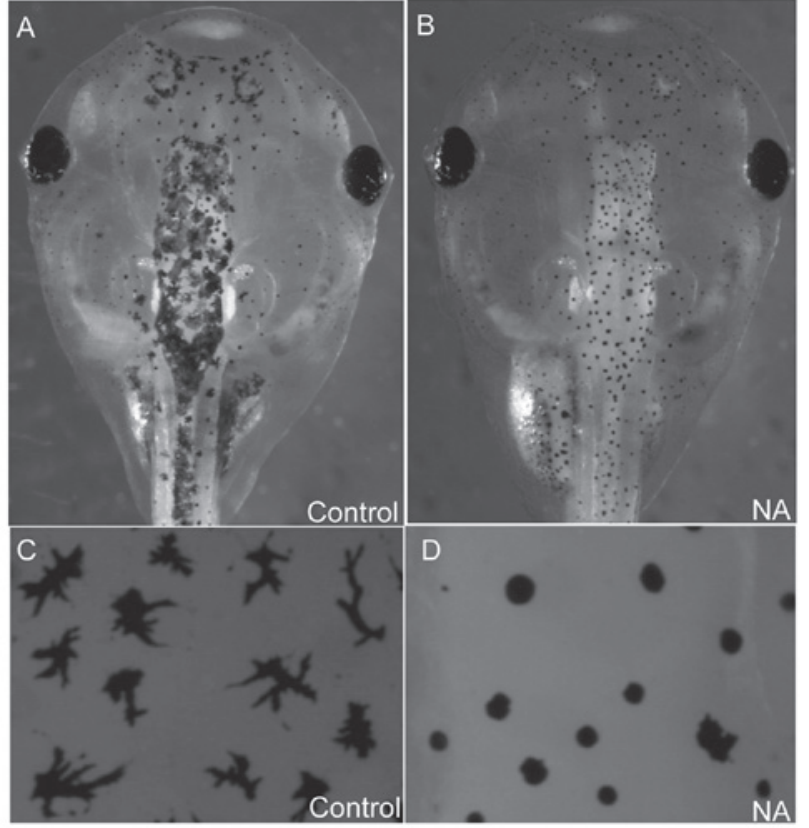

Figure 5. Microinjection of $70 \mathrm{ng}$ NA into xenopus embryos inhibited the melanosome transport. NA, nicotinic acid. (Magnification, x30).

Abnormal increases in the $\mathrm{Ca}^{2+}$ concentration contribute to the disassembly of F-actin. To further elucidate the association between changes in $\left[\mathrm{Ca}^{2+}\right]$ and the disassembly of the cytoskeleton, a $\mathrm{CaCl}_{2}$ solution was added directly into the NIH3T3 cell culture media and the cytoskeleton structure was examined following $1 \mathrm{~h}$ of incubation. Consistent with the effect of NA demonstrated above, $40 \mathrm{mM} \mathrm{CaCl}{ }_{2}$ disrupted F-actin filaments (Fig. 3A) into punctuate G-actin 
spots (Fig. 3D). However, artificial increases in $\left[\mathrm{Ca}^{2+}\right]$ did not affect the microtubular structure (Fig. 3E) compared with that of the control group (Fig. 3B). The results implied that the $\mathrm{Ca}^{2+}$ wave induced by NA may be involved in the disruption of F-actin filaments, but not in the disassembly of the microtubular polymer structure.

Depolymerized microtubule subunits undergo ubiquitin-proteasome degradation. Microtubules consist of $\alpha$-tubulin and $\beta$-tubulin hetero-subunits. The microtubules were labeled with anti- $\beta$ tubulin antibody. Under exposure to $70 \mathrm{mM}$ NA for $1 \mathrm{~h}$, not only did the microtubule-stained pattern change, but also its immunofluorescent intensity decreased significantly (Fig. 2J, M and O). To confirm these results, a total amount of $\beta$ tubulin was analyzed using western blot analysis. As expected, NA markedly downregulated $\beta$-tubulin at the protein level. In addition, MG132, an inhibitor of the ubiquitin-proteasome pathway, was able to reverse $\beta$-tubulin reduction (Fig. 4). However, there were no significant changes in $\mathrm{F}$-actin monomer protein $\mathrm{G}$-actin (data not shown).

NA blocks melanosome intracellular transport in xenopus embryos. In the cultured cells NA disrupted cytoskeletal integrity and may have inhibited intracellular trafficking. To investigate the effect of NA on intracellular transport processes in vivo, 70 ng NA was microinjected into xenopus embryos and its effect on melanosome transport in melanocytes was observed. Melanosomes either disperse or aggregate along microtubules and F actin-filaments (18). As they are easy to observe, melanocytes represent a reliable system for investigating intracellular transport. In normal embryos, the melanosomes disperse uniformly in a dendritic type manner (Fig. 5A and C). By contrast, in NA-microinjected embryos, melanosome transport was blocked and exhibited an aggregated disc type morphology (Fig. 5B and D), suggesting that NA blocked intracellular transport processes in vivo.

\section{Discussion}

Previous studies have demonstrated that $100 \mu \mathrm{M}$ NA evokes intracellular $\left[\mathrm{Ca}^{2+}\right]$ within several minutes (8-10); however, the detailed mechanisms underlying this effect remains elusive. In the present study, intracellular $\left[\mathrm{Ca}^{2+}\right]$ was assessed in a time lapse manner upon exposure to NA. NIH3T3 cells required higher quantities of NA to evoke any effects on intracellular $\mathrm{Ca}^{2+}$. As expected, the first response of NIH3T3 cells to NA was not an elevation but a reduction in intracellular $\left[\mathrm{Ca}^{2+}\right]$. The $\left[\mathrm{Ca}^{2+}\right]$ increase following $\left[\mathrm{Ca}^{2+}\right]$ reduction may be disrupted by ruining $\mathrm{Ca}^{2+}$ storage in the ER by TG, an endoplasmic reticulum $\mathrm{Ca}^{2+}$-ATPase pump inhibitor. Therefore, it was suggested that the overall effect of $\left[\mathrm{Ca}^{2+}\right]$ increase may be divided into two steps. Firstly, NA reduces intracellular $\left[\mathrm{Ca}^{2+}\right]$, possibly via triggering the efflux of $\mathrm{Ca}^{2+}$ ions out of the cell membrane channels. Secondly, the $\mathrm{Ca}^{2+}$ release from the ER contributes at least in part to the $\left[\mathrm{Ca}^{2+}\right]$ elevation. Since small amounts of NA $(1 \mathrm{mM})$ do not elevate but only reduce $\left[\mathrm{Ca}^{2+}\right]$, the release of $\mathrm{Ca}^{2+}$ from the ER requires higher concentrations of NA to trigger this process.
The present study sought to elucidate the mechanisms underlying NA-induced changes in intracellular $\left[\mathrm{Ca}^{2+}\right]$ waves. Based on other studies and the data of the present study, a hypothesis was proposed that the metabolism of NA adenine dinucleotide phosphate (NAADP) is important during NA modulation of cellular $\mathrm{Ca}^{2+}$. A putative synthesis pathway for NAADP exists, NAADP is a well established $\mathrm{Ca}^{2+}$ mobilizing agent that releases $\mathrm{Ca}^{2+}$ from intracellular stores (19). In the presence of NA and nicotinamide adenine dinucleotide phosphate (NADP), ADP-ribosyl cyclase catalyzes the synthesis of NAADP by a base exchange reaction and cAMP is a stimulator during this process (20-22). Notably, NA reduces the intracellular cAMP concentration (5). At a high concentration, NA may inhibit cAMP and thereby limit the synthesis of NAADP. The decrease of NAADP may be responsible for the first $\left[\mathrm{Ca}^{2+}\right]$ drop upon exposure to NA. Excessive NA may rapidly overcome the cAMP-limited step and promote the synthesis of NAADP. Therefore, a marked $\left[\mathrm{Ca}^{2+}\right]$ elevation was observed. Furthermore, a markedly high concentration of NA (100 mM) may completely eradicate cAMP and re-establish cAMP as a rate-limiting step. Therefore, the $\left[\mathrm{Ca}^{2+}\right]$ elevation curve following $100 \mathrm{mM}$ NA treatment is not as steep as that following $10 \mathrm{mM}$ NA treatment. In the present study, $2 \mathrm{mM}$ 8-Br-cAMP (a cAMP analog) delayed and alleviated the first $\left[\mathrm{Ca}^{2+}\right]$ drop in response to NA, suggesting that cAMP has a key role in the changes in the $\left[\mathrm{Ca}^{2+}\right]$ wave induced by NA.

It is well established that the intracellular $\mathrm{Ca}^{2+}$ wave may modulate the cytoskeletal structure (23-28). Although F-actins and microtubules underwent disassembly upon incubation with $\mathrm{NA}$, the external addition of high concentrations of $\mathrm{CaCl}_{2}$ only disrupted the $\mathrm{F}$-actin filaments. It was hypothesized that besides the $\left[\mathrm{Ca}^{2+}\right]$ elevation, other pathways must also be involved in the disassembly of microtubules. The disruption of F-actin and microtubule cytoskeleton may definitely negatively effect the intracellular traffic process. In cultured cells, an opaque material accumulated around the nucleus when incubated with $70 \mathrm{mM}$ NA. It appears that the minus end (nuclear region) to plus end (cell membrane region)-directed transport process was inhibited and therefore, cargo was deposited in the perinuclear region. Further evidence in the xenopus melanocyte system confirmed that NA induced an intracytic transport deficiency.

In conclusion, the present study showed that NA regulated the intracellular calcium concentration depending on its initial concentration and exposure time. High concentrations of nicotinic acid induced cytoskeletal disassembly and promoted $\beta$-tubulin degradation in a proteasome-dependent manner. The cytoskeletal disassembly may finally contribute to the disruption of the intracellular transport process. Further investigations aim to minimize the functional concentration of NA and characterize the function of NA in different biological systems, particularly in cancer cells and animal models. As the cytoskeleton is essential during cell migration and EMT, interrupting the dynamic arrangement of the cytoskeletion may break the fundamental cancerous processes of metastasis. NA provides potential for clinical use in the future.

\section{Acknowledgements}

The authors are grateful to Dr Bingyu Mao for providing the experimental reagents. This study was supported by the 
National Natural Science Foundation of China (grant nos. 81102519 to Y.S. and 81200878 to J.L.), the China Postdoctoral Science Foundation funded project (no. 2012M511914 to Y.S.), and the Chongqing Science and Technology Committee (no. cstc2012jjA0147 to Y.S.). The funders had no role in the study design, data collection and analysis, decision to publish or preparation of the manuscript.

\section{References}

1. Altschul R, Hoffer A and Stephen JD: Influence of nicotinic acid on serum cholesterol in man. Arch Biochem Biophys 54: 558-559, 1955.

2. Carlson LA: Nicotinic acid: the broad-spectrum lipid drug. A 50th anniversary review. J Intern Med 258: 94-114, 2005.

3. Figge HL, Figge J, Souney PF, et al: Nicotinic acid: a review of its clinical use in the treatment of lipid disorders. J Pharm Pharmacol 8: 287-294, 1988.

4. Offermanns S: The nicotinic acid receptor GPR109A (HM74A or PUMA-G) as a new therapeutic target. Trends Pharmacol Sci 27: 384-390, 2006.

5. Gille A, Bodor ET, Ahmed K, et al: Nicotinic acid: pharmacological effects and mechanisms of action. Annu Rev Pharmacol Toxicol 48: 79-106, 2008.

6. Morrow JD, Parsons WG IIIrd and Roberts LJ IInd: Release of markedly increased quantities of prostaglandin D2 in vivo in humans following the administration of nicotinic acid. Prostaglandins 38: 263-274, 1989.

7. Andersson RGG, Aberg G, Brattsand R, et al: Studies on the mechanism of flush induced by nicotinic acid. Acta Pharmacol Toxicol (Copenh) 41: 1-10, 1977.

8. Benyó Z, Gille A, Kero J, et al: GPR109A (PUMA-G/HM74A) mediates nicotinic acid-induced flushing. J Clin Invest 115 3634-3640, 2005.

9. Tunaru S, Kero J, Schaub A, et al: PUMA-G and HM74 are receptors for nicotinic acid and mediate its anti-lipolytic effect. Nat Med 9: 352-355, 2003.

10. Kostylina G, Simon D, Fey MF, et al: Neutrophil apoptosis mediated by nicotinic acid receptors (GPR109A). Cell Death Differ 15: 134-142, 2008

11. Zhao S, Jiang H, Wang W, et al: Cloning and developmental expression of the Xenopus Nkx6 genes. Dev Genes Evol 217: 477-483, 2007.

12. Shi Y, Zhao S, Li J, et al: Islet-1 is required for ventral neuron survival in Xenopus. Biochem Biophys Res Commun 388: 506-510, 2009.

13. Downey GP, Chan CK, Trudel S, et al: Actin assembly in electropermeabilized neutrophils: role of intracellular calcium. J Cell Biol 110: 1975-1982, 1990.
14. Yoneda M, Nishizaki T, Tasaka K, et al: Changes in actin network during calcium-induced exocytosis in permeabilized GH3 cells: calcium directly regulates F-actin disassembly. J Endocrinol 166: 677-687, 2000.

15. Forscher P: Calcium and polyphosphoinositide control of cytoskeletal dynamics. Trends Neurosci 12: 468-474, 1989.

16. Rosado JA and Sage SO: The actin cytoskeleton in store-mediated calcium entry. J Physiol 526: 221-229, 2000.

17. Wilson MT, Kisaalita WS and Keith CH: Glutamate-induced changes in the pattern of hippocampal dendrite outgrowth: a role for calcium-dependent pathways and the microtubule cytoskeleton. J Neurobiol 43: 159-172, 2000.

18. Sheets L, Ransom DG, Mellgren EM, et al: Zebrafish melanophilin facilitates melanosome dispersion by regulating dynein. Curr Biol 17: 1721-1734, 2007.

19. Yamasaki M, Churchill GC and Galione A: Calcium signalling by nicotinic acid adenine dinucleotide phosphate (NAADP). FEBS J 272: 4598-4606, 2005.

20. Aarhus R, Graeff RM, Dickey DM, et al: ADP-ribosyl cyclase and CD38 catalyze the synthesis of a calcium-mobilizing metabolite from NADP. J Biol Chem 270: 30327-30333, 1995.

21. Wilson $\mathrm{H}$ and Galione A: Differential regulation of nicotinic acid-adenine dinucleotide phosphate and cADP-ribose production by cAMP and cGMP. Biochem J 331: 837-843, 1998.

22. Rah SY, Mushtaq M, Nam TS, et al: Generation of cyclic ADP-ribose and nicotinic acid adenine dinucleotide phosphate by $\mathrm{CD} 38$ for $\mathrm{Ca} 2+$ signaling in interleukin-8-treated lymphokine-activated killer cells. J Biol Chem 285: 21877-21887, 2010.

23. Sobue K, Kanda K, Adachi J, et al: Calmodulin-binding proteins that interact with actin filaments in a $\mathrm{Ca}^{2+}$-dependent flip-flop manner: survey in brain and secretory tissues. Proc Natl Acad Sci USA 80: 6868-6871, 1983.

24. Shin DM, Zhao XS, Zeng W, et al: The mammalian Sec6/8 complex interacts with $\mathrm{Ca}^{2+}$ signaling complexes and regulates their activity. J Cell Biol 150: 1101-1112, 2000.

25. Constantin B, Meerschaert K, Vandekerckhove J, et al: Disruption of the actin cytoskeleton of mammalian cells by the capping complex actin-fragmin is inhibited by actin phosphorylation and regulated by $\mathrm{Ca}^{2+}$ ions. J Cell Sci 111: 1695-1706, 1998.

26. Brown SS, Yamamoto K and Spudich JA: A 40,000-dalton protein from Dictyostelium discoideum affects assembly properties of actin in a $\mathrm{Ca}^{2+}$-dependent manner. J Cell Biol 93: 205-210, 1982.

27. Yamamoto H, Fukunaga K, Tanaka E, et al: $\mathrm{Ca}^{2+}$ - and calmodulin-dependent phosphorylation of microtubule-associated protein 2 and tau factor, and inhibition of microtubule assembly. J Neurochem 41: 1119-1125, 1983.

28. Gradin HM, Marklund U, Larsson N, et al: Regulation of microtubule dynamics by $\mathrm{Ca}^{2+} /$ calmodulin-dependent kinase IV/Gr-dependent phosphorylation of oncoprotein 18. Mol Cell Biol 17: 3459-3467, 1997. 